\title{
New inequalities between the inverse hyperbolic tangent and the analogue for corresponding functions
}

\author{
Xiao-Diao Chen ${ }^{1 *}$, Long $\mathrm{Nie}^{1}$ and Wangkang Huang ${ }^{1}$
}

\section{"Correspondence:}

xiaodiao@hdu.edu.cn

'Key Laboratory of Complex

Systems Modeling and Simulation,

Hangzhou Dianzi University,

Hangzhou, China

\begin{abstract}
In this paper, we present new inequalities which reveal further relationship for both the inverse tangent function $\arctan (x)$ and the inverse hyperbolic function $\operatorname{arctanh}(x)$. At the same time, we give the analogue for inverse hyperbolic tangent and other corresponding functions.
\end{abstract}

Keywords: Inequalities; Inverse tangent function; Inverse hyperbolic sine function; Inverse hyperbolic tangent function; Inverse sine function

\section{Introduction}

Masjed-Jamei [1] obtained the following inequality for $|x|<1$ :

$$
(\arctan x)^{2} \leq \frac{x \ln \left(x+\sqrt{1+x^{2}}\right)}{\sqrt{1+x^{2}}} .
$$

Many similar or relative inequalities are discussed in references [2-14]. Recently, Zhu and Malesevic [15] affirmed inequality (1) for the large interval $(-\infty, \infty)$, pointed out that $\sinh ^{-1}(x)=\ln \left(x+\sqrt{1+x^{2}}\right)$, and provided the following Theorems 1-6, which (or relative results) can be also found in $[11,12]$.

Theorem 1 ([15]) The inequality

$$
(\arctan x)^{2} \leq \frac{x \ln \left(x+\sqrt{1+x^{2}}\right)}{\sqrt{1+x^{2}}}
$$

holds for all $x \in(-\infty, \infty)$, and the power number 2 is the best in (2).

Theorem 2 ([15]) Let $0<r<\infty, \lambda=1$, and $\mu=r \ln \left(r+\sqrt{r^{2}+1}\right) /\left(\sqrt{r^{2}+1}(\arctan r)^{2}\right)$. Then the double inequality

$$
\lambda(\arctan x)^{2} \leq \frac{x \ln \left(x+\sqrt{1+x^{2}}\right)}{\sqrt{1+x^{2}}} \leq \mu(\arctan x)^{2}
$$

holds for all $x \in(--r, r)$, where $\lambda$ and $\mu$ are the best constants in (3).

(c) The Author(s) 2020. This article is licensed under a Creative Commons Attribution 4.0 International License, which permits use, sharing, adaptation, distribution and reproduction in any medium or format, as long as you give appropriate credit to the original author(s) and the source, provide a link to the Creative Commons licence, and indicate if changes were made. The images or other third party material in this article are included in the article's Creative Commons licence, unless indicated otherwise in a credit line to the material. If material is not included in the article's Creative Commons licence and your intended use is not permitted by statutory regulation or exceeds the permitted use, you will need to obtain permission directly from the copyright holder. To view a copy of this licence, visit http://creativecommons.org/licenses/by/4.0/. 
Theorem 3 ([15]) We have

$$
\begin{aligned}
-\frac{1}{45} x^{6} \leq(\arctan x)^{2}-\frac{x \ln \left(x+\sqrt{1+x^{2}}\right)}{\sqrt{1+x^{2}}} & \leq-\frac{1}{45} x^{6}+\frac{4}{105} x^{8} \\
-\frac{1}{45} x^{6}+\frac{4}{105} x^{8}-\frac{11}{225} x^{10} & \leq(\arctan x)^{2}-\frac{x \ln \left(x+\sqrt{1+x^{2}}\right)}{\sqrt{1+x^{2}}} \\
& \leq-\frac{1}{45} x^{6} \leq+\frac{4}{105} x^{8}-\frac{11}{225} x^{10}+\frac{586}{10,395} x^{12} .
\end{aligned}
$$

Theorem 4 ([15]) The inequality

$$
(\operatorname{arctanh} x)^{2} \leq \frac{x \arcsin x}{\sqrt{1-x^{2}}}
$$

holds for all $x \in(-1,1)$, and the power number 2 is the best in (6).

Theorem 5 ([15]) Let $0<r<1, \alpha_{1}=1$, and $\beta_{1}=r(\arcsin r) /\left(\sqrt{1--r^{2}}(\operatorname{arctanh} r)^{2}\right)$. Then the double inequality

$$
\alpha_{1}(\operatorname{arctanh} x)^{2} \leq \frac{x \arcsin x}{\sqrt{1-x^{2}}} \leq \beta_{1}(\operatorname{arctanh} x)^{2}
$$

holds for all $x \in(-r, r)$, where $\alpha_{1}$ and $\beta_{1}$ are the best constants in (7).

Recently, Chen and Malešević [14] proposed the following results:

$$
\begin{aligned}
& \frac{x \operatorname{arcsinh} x}{\sqrt{1+x^{2}+\alpha_{2} x^{4}}} \leq(\arctan x)^{2} \leq \frac{x \operatorname{arcsinh} x}{\sqrt{1+x^{2}+\beta_{2} x^{4}}}, \quad x>0, \\
& \frac{x \arcsin x}{1-\alpha_{3} x^{2}}<(\operatorname{arctanh} x)^{2}, \quad 0<x<1,
\end{aligned}
$$

where $\alpha_{2}=\frac{2}{45}, \beta_{2}=0$, and $\alpha_{3}=\frac{1}{2}$ are the best possible constants.

In 2020, Zhu and Malešević [13] proposed natural approximation of Masjed-Jamei's inequality and provided two-sided bounds in a polynomial form of $(\arctan x)^{2}-\frac{x \ln \left(x+\sqrt{1+x^{2}}\right)}{\sqrt{1+x^{2}}}$, which consists of explicit formulae of different degrees.

The values of $\mu$ in Theorem 2 and $\beta_{1}$ in Theorem 5 tend to be $+\infty$ for $r$ tends to be $\pm \infty$ and \pm 1 , respectively. In this paper, we obtain the following new inequalities, which improve the approximation effect of the inequalities in [15]. The main results are as follows.

Theorem 6 The inequality

$$
(\arctan x)^{2} \geq \frac{3\left(8+9 x^{2}-8 \sqrt{1+x^{2}}\right)}{\left(4+11 \sqrt{1+x^{2}}\right) \sqrt{1+x^{2}}} \triangleq F(x)
$$

holds for all $x \in(-\infty, \infty)$. 
Theorem 7 Let $\kappa_{1}=\frac{108}{11 \pi^{2}} \approx 0.9947$ and $\kappa_{2}=1$. The inequality

$$
\kappa_{1}(\arctan x)^{2} \leq F(x) \leq \kappa_{2}(\arctan x)^{2}
$$

holds for all $x \in(-\infty, \infty)$, where $\kappa_{1}$ and $\kappa_{2}$ are the best constants in (11).

Theorem 8 The inequality

$$
\frac{23}{75,600} x^{8} \geq(\arctan x)^{2}-F(x) \geq \frac{23}{75,600} x^{8}-\frac{899}{1,134,000} x^{10}
$$

holds for all $x \in(-\infty, \infty)$.

Theorem 9 The inequality

$$
G_{1}(x) \triangleq(\operatorname{arctanh} x)^{2} \leq\left(\frac{-\ln \left(1-x^{2}\right)}{\arcsin x}\right)^{2} \triangleq G_{2}(x)
$$

holds for all $x \in(-1,1)$.

Theorem 10 Let $\kappa_{3}=1$ and $\kappa_{4}=\frac{16}{\pi^{2}} \approx 1.6211$. The inequality

$$
\kappa_{3}(\operatorname{arctanh} x)^{2} \leq\left(\frac{-\ln \left(1-x^{2}\right)}{\arcsin x}\right)^{2} \leq \kappa_{4}(\operatorname{arctanh} x)^{2}
$$

holds for all $x \in(-1,1)$, where $\kappa_{3}$ and $\kappa_{4}$ are the best constants in (14).

\section{Proofs of Theorems 6-10}

Let $\arctan x=t$, then one has that $x=\tan (t)$ and $\sqrt{1+x^{2}}=\sec (t)$, where $x \in(-\infty, \infty)$ and $t \in(-\pi / 2, \pi / 2)$. It can be verified that

$$
\begin{aligned}
& (\arctan x)^{2}=t^{2} \\
& F(x)=-\frac{3}{4} \cos (t)-\frac{63}{16}+\frac{1125}{16(4 \cos (t)+11)}=f_{1}(t) \\
& (\arctan x)^{2}-F(x)=\left(t^{2}-f_{1}(t)\right)=\delta_{1}(t), \\
& \delta_{1}^{\prime \prime \prime}(t)=\frac{\left(12\left(16 \cos (t)^{2}+208 \cos (t)+1501\right)\right)(\cos (t)-1)^{2} \sin (t)}{(4 \cos (t)+11)^{4}} .
\end{aligned}
$$

\subsection{Proof of Theorem 6}

From Eq. (15), one has that

$$
\delta_{1}^{\prime \prime \prime}(t)>0, \quad t \in(0, \pi / 2), \quad \delta_{1}^{\prime \prime}(0)=\delta_{1}^{\prime}(0)=\delta_{1}(0)=0,
$$

which leads to

$$
\delta_{1}^{\prime \prime}(t)>0, \quad \delta_{1}^{\prime}(t)>0, \quad t \in(0, \pi / 2), \quad \delta_{1}(t) \geq \delta_{1}(0)=0, \quad t \in[0, \pi / 2) .
$$


Note that $\delta_{1}(t)=\delta_{1}(-t)$, combining Eq. (15) with Eq. (17), one has that

$$
\delta_{1}(t) \geq 0, \quad t \in(-\pi / 2, \pi / 2), \quad \text { and } \quad(\arctan x)^{2}-F(x) \geq 0, \quad x \in(-\infty, \infty) \text {. }
$$

And we complete the proof.

\subsection{Proof of Theorem 7}

From Theorem 6, one has that

$$
F(x) \leq \kappa_{2}(\arctan x)^{2}
$$

Now we prove that $\kappa_{1}(\arctan x)^{2} \leq F(x)$. From Eq. (15), one has that

$$
\begin{aligned}
& \kappa_{1}(\arctan x)^{2}-F(x)=\kappa_{1} t^{2}-f_{1}(t)=\delta_{2}(t), \\
& \delta_{2}^{\prime \prime \prime}(t)=-f_{1}^{\prime \prime \prime}(t)=\delta_{1}^{\prime \prime \prime}(t)>0, \quad t \in(0, \pi / 2), \\
& \delta_{2}^{\prime \prime}(0)=\frac{216-22 \pi^{2}}{11 \pi^{2}} \approx-0.01<0, \quad \delta_{2}^{\prime \prime}(\pi / 2)=\frac{26136-2250 \pi^{2}}{1331 \pi^{2}} \approx 0.2>0 .
\end{aligned}
$$

From Eq. (19), there exists a unique root $t_{1} \in(0, \pi / 2)$ such that

$$
\begin{aligned}
& \delta_{2}^{\prime \prime}(t)<0, \quad t \in\left(0, t_{1}\right), \quad \delta_{2}^{\prime}(0)=0, \\
& \delta_{2}^{\prime \prime}(t)>0, \quad t \in\left(t_{1}, \pi / 2\right), \quad \delta_{2}^{\prime}(\pi / 2)=\frac{1188-372 \pi}{121 \pi} \approx 0.05>0 .
\end{aligned}
$$

From Eq. (19), there exists a unique root $t_{2} \in\left(t_{1}, \pi / 2\right)$ such that

$$
\begin{aligned}
& \delta_{2}^{\prime}(t)<0, \quad t \in\left(0, t_{2}\right), \quad \delta_{2}(0)=0, \\
& \delta_{2}^{\prime}(t)>0, \quad t \in\left(t_{2}, \pi / 2\right), \quad \delta_{2}(\pi / 2)=0 .
\end{aligned}
$$

From Eq. (21), one has that

$$
\delta_{2}(t) \leq 0, \quad t \in\left[0, t_{2}\right] \cup\left[t_{2}, \pi / 2\right)=[0, \pi / 2) .
$$

Note that $\delta_{2}(t)=\delta_{2}(-t)$, combining Eq. (19) with Eq. (22), one has that

$$
\delta_{2}(t) \leq 0, \quad t \in(-\pi / 2, \pi / 2), \quad \text { and } \quad \kappa_{1}(\arctan x)^{2} \leq F(x), \quad x \in(-\infty, \infty) .
$$

Note that

$$
\lim _{x \rightarrow \infty} \frac{F(x)}{(\arctan x)^{2}}=\kappa_{1}, \quad \lim _{x \rightarrow 0} \frac{F(x)}{(\arctan x)^{2}}=\kappa_{2}
$$

both $\kappa_{1}$ and $\kappa_{2}$ are the best constants. And the proof is completed. 


\subsection{Proof of Theorem 8}

Let $f_{2}(t)=\frac{23}{75,600}(\tan t)^{8}$ and $f_{3}(t)=\frac{23}{75,600}(\tan t)^{8}-\frac{899}{1,134,000}(\tan t)^{10}$. Equation (12) in Theorem 8 is equivalent to

$$
\delta_{3}(t)=\delta_{1}(t)-f_{2}(t) \leq 0, \quad \delta_{4}(t)=\delta_{1}(t)-f_{3}(t) \geq 0, \quad t \in(-\pi / 2, \pi / 2) .
$$

It can be verified that

$$
\begin{aligned}
f_{2}^{\prime \prime \prime}(t) & =\frac{23 \sin (t)^{5}\left(2 \cos (t)^{4}-26 \cos (t)^{2}+45\right)}{4725(\cos t)^{11}} \\
f_{3}^{\prime \prime \prime}(t) & =\frac{\sin (t)^{5}\left(1175 \cos (t)^{6}-18871 \cos (t)^{4}+50,261 \cos (t)^{2}-29,667\right)}{28,350(\cos t)^{13}} .
\end{aligned}
$$

Let $\phi_{1}(t)=907,200 \cos (t)^{12}+12,700,800 \cos (t)^{11}+97,807,500 \cos (t)^{10}+97,795,724 \cos (t)^{9}+$ $97,642,636 \cos (t)^{8}+96,990,540 \cos (t)^{7}+96,802,860 \cos (t)^{6}+103,838,238 \cos (t)^{5}+$ $126,378,882 \cos (t)^{4}+148,760,458 \cos (t)^{3}+130,005,062 \cos (t)^{2}+67,501,665 \cos (t)+$ $15,153,435$ and $\phi_{2}(t)=5,443,200 \cos (t)^{13}+81,648,000 \cos (t)^{12}+668,493,000 \cos (t)^{11}+$ $1,255,037,200 \cos (t)^{10}+1,837,671,000 \cos (t)^{9}+2,404,568,576 \cos (t)^{8}+$ $2,978,639,640 \cos (t)^{7}+3,789,264,297 \cos (t)^{6}+5,266,619,820 \cos (t)^{5}+$ $7,153,847,855 \cos (t)^{4}+7,714,708,320 \cos (t)^{3}+5,610,730,675 \cos (t)^{2}+2,369,206,620 \cos (t)+$ 434,354,547. Combining Eq. (24) with Eq. (25), one has that

$$
\begin{aligned}
& \delta_{3}^{\prime \prime \prime}(t)=\frac{\sin (t)(\cos (t)-1)^{3}}{(4 \cos (t)+11)^{4}(\cos t)^{11}} \phi_{1}(t)<0, \quad \forall t \in(0, \pi / 2), \\
& \delta_{4}^{\prime \prime \prime}(t)=\frac{\sin (t)(\cos (t)-1)^{4}}{28,350(4 \cos (t)+11)^{4}(\cos t)^{13}} \phi_{2}(t)>0, \quad \forall t \in(0, \pi / 2), \\
& \delta_{3}^{\prime \prime}(0)=0, \quad \delta_{4}^{\prime \prime}(0)=0 .
\end{aligned}
$$

From Eq. (25), one has that

$$
\delta_{3}^{\prime \prime}(t)<0, \quad \delta_{4}^{\prime \prime}(t)>0, \quad \forall t \in(0, \pi / 2), \quad \delta_{3}^{\prime}(0)=0, \quad \delta_{4}^{\prime}(0)=0 .
$$

From Eq. (27), one obtains that

$$
\delta_{3}^{\prime}(t)<0, \quad \delta_{4}^{\prime}(t)>0, \quad \forall t \in(0, \pi / 2), \quad \delta_{3}(0)=0, \quad \delta_{4}(0)=0,
$$

which leads to

$$
\delta_{3}(t) \leq 0, \quad \delta_{4}(t) \geq 0, \quad \forall t \in[0, \pi / 2)
$$

Note that $\delta_{i}(t)=\delta_{i}(-t), i=3,4$, combining with Eq. (29), both Eq. (24) and Theorem 8 are proved. 


\subsection{Proof of Theorem 9}

Let $\arcsin (x)=s$, then one has that $x=\sin (s)$, where $x \in(-1,1), s \in(-\pi / 2, \pi / 2)$. It can be verified that

$$
\begin{aligned}
& (\operatorname{arctanh} x)=\frac{1}{2} \ln \left(\frac{1+\sin (s)}{1-\sin (s)}\right)>0, \\
& \left(\frac{-\ln \left(1-x^{2}\right)}{\arcsin x}\right)=\frac{-\ln \left(1-(\sin s)^{2}\right)}{s}>0, \quad s \in(0, \pi / 2) .
\end{aligned}
$$

Let

$$
\begin{aligned}
& (\operatorname{arctanh} x)-\left(\frac{-\ln \left(1-x^{2}\right)}{\arcsin x}\right)=\frac{1}{2} \ln \left(\frac{1+\sin (s)}{1-\sin (s)}\right)-\frac{-\ln \left(1-(\sin s)^{2}\right)}{s}=\delta_{5}(s), \\
& \delta_{6}(s)=\delta_{5}^{\prime}(s) \cdot s^{2}, \quad \phi_{3}(s)=-2+\sin (s) s+2 \cos (s) .
\end{aligned}
$$

It can be verified that

$$
\phi_{3}^{\prime \prime}(s)=-\sin (s) s<0, \quad s \in(0, \pi / 2), \quad \phi_{3}^{\prime}(0)=\phi_{3}(0)=0
$$

which leads to

$$
\phi_{3}(s) \leq 0, \quad \delta_{6}^{\prime}(s)=\frac{s}{(\cos s)^{2}} \phi_{3}(s) \leq 0, \quad \delta_{6}(0)=0, \quad s \in[0, \pi / 2) .
$$

Combining Eq. (31) with Eq. (32), one obtains that

$$
\delta_{6}(s) \leq 0, \quad \delta_{5}^{\prime}(s) \leq 0, \quad \delta_{5}(0)=0, \quad s \in[0, \pi / 2) .
$$

Combining Eq. (31) with Eq. (33), we have that

$$
\delta_{5}(s) \leq 0, \quad s \in[0, \pi / 2), \quad 0 \leq(\operatorname{arctanh} x)^{2} \leq\left(\frac{-\ln \left(1-x^{2}\right)}{\arcsin x}\right)^{2}, \quad x \in[0,1) .
$$

Note that $G_{i}(-x)=G_{i}(x), i=1,2$, combining with Eq. (34), we have proved both Eq. (13) and Theorem 9 .

\subsection{Proof of Theorem 10}

Directly from Theorem 9, we have proved the left-hand side in Eq. (14) in Theorem 10.

$$
\kappa_{3}(\operatorname{arctanh} x)^{2} \leq\left(\frac{-\ln \left(1-x^{2}\right)}{\arcsin x}\right)^{2}
$$

Now, we will prove the right-hand side of Eq. (14). Combining with Eq. (30), let

$$
\begin{aligned}
& \frac{4}{\pi}(\operatorname{arctanh} x)-\left(\frac{-\ln \left(1-x^{2}\right)}{\arcsin x}\right)=\frac{4}{2 \pi} \ln \left(\frac{1+\sin (s)}{1-\sin (s)}\right)-\frac{-\ln \left(1-(\sin s)^{2}\right)}{s} \triangleq \delta_{7}(s), \\
& \delta_{8}(s)=\delta_{7}^{\prime}(s) \cdot s^{2}, \quad \phi_{4}(s)=\frac{2(2 \sin (s) s+4 \cos (s)-\pi)}{\pi} .
\end{aligned}
$$


It can be verified that

$$
\phi_{4}^{\prime \prime}(s)=\frac{-4 \sin (s) s}{\pi}<0, \quad s \in(0, \pi / 2), \quad \phi_{4}^{\prime}(0)=\phi_{4}(\pi / 2)=0,
$$

which leads to

$$
\phi_{4}(s) \geq 0, \quad \delta_{8}^{\prime}(s)=\frac{s}{(\cos s)^{2}} \phi_{4}(s) \geq 0, \quad \delta_{8}(0)=0, \quad s \in[0, \pi / 2) .
$$

Combining Eq. (35) with Eq. (36), one obtains that

$$
\delta_{8}(s) \geq 0, \quad \delta_{7}^{\prime}(s) \geq 0, \quad \delta_{7}(0)=0, \quad s \in[0, \pi / 2) .
$$

Combining Eq. (35) with Eq. (37), we have that

$$
\begin{aligned}
& \delta_{7}(s) \geq 0, \quad s \in[0, \pi / 2), \\
& 0 \leq\left(\frac{-\ln \left(1-x^{2}\right)}{\arcsin x}\right)^{2} \leq\left(\frac{4}{\pi} \operatorname{arctanh} x\right)^{2}, \quad x \in[0,1) .
\end{aligned}
$$

Note that $G_{i}(-x)=G_{i}(x), i=1,2$, combining with Eq. (38), one obtains that

$$
\left(\frac{-\ln \left(1-x^{2}\right)}{\arcsin x}\right)^{2} \leq \kappa_{2}(\operatorname{arctanh} x)^{2}, \quad x \in(-1,1) .
$$

Combining Theorem 9 with Eq. (39), we have completed the proofs of both Eq. (14) and Theorem 10.

\section{Discussions and conclusions}

The values of $\mu$ in Theorem 2 and $\beta_{1}$ in Theorem 5 tend to be $+\infty$ for $r$ tends to be $\pm \infty$ and \pm 1 , respectively, while the values of $\kappa_{i}$ in Theorems 7 and 10 are constant. The error plots of the bounds from Eq. (2) and Eq. (6) in [15], from Eq. (8) and Eq. (9) in [14], and from Eq. (6) and Eq. (13) are plotted in Fig. 1. It shows that the results of Eq. (11) and

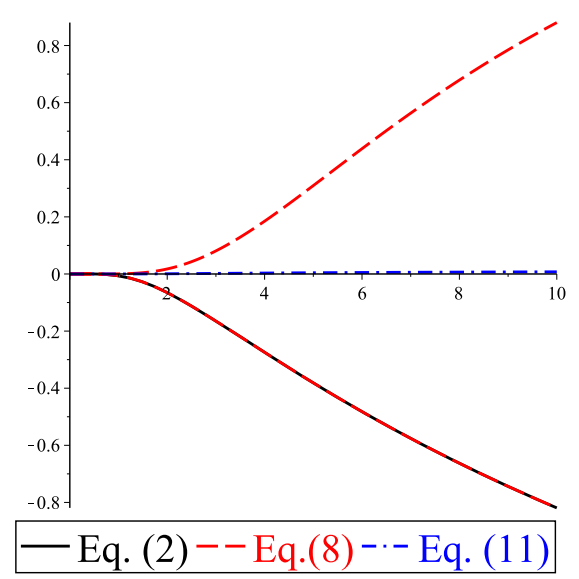

(a)

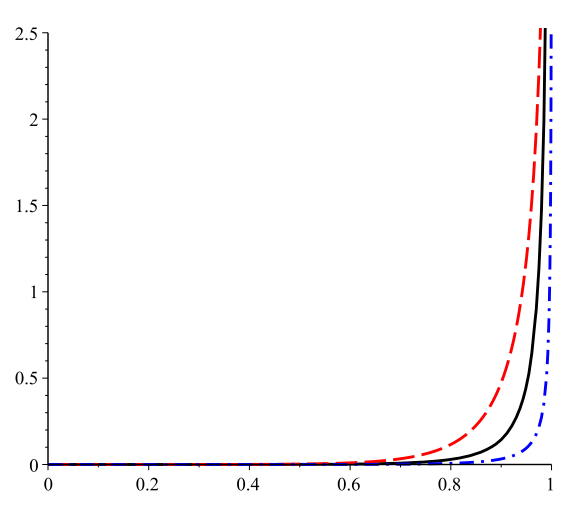

Eq. (6) -- Eq. (9) ${ }^{-\cdot-}$ Eq. (13)

Figure 1 Error plots of bounds from (a) Eq. (2), (8), and (11); and (b) Eq. (6), (9), and (13) 
Eq. (13) in this paper achieve better approximation effect than those of Eq. (2), Eq. (6), Eq. (8), and Eq. (9).

\author{
Acknowledgements \\ The authors would like to thank the editor and the anonymous referees for their valuable suggestions and comments \\ which helped us to improve this paper greatly.
}

\title{
Funding
}

This research work was partially supported by Zhejiang Key Research and Development Project of China (LY19F020041, 2018C01030), the National Science Foundation of China (61972120,61672009).

\section{Availability of data and materials}

Not applicable.

\section{Competing interests}

The authors declare that they have no competing interests.

\section{Authors' contributions}

All authors contributed equally to the manuscript and read and approved the final manuscript.

\section{Publisher's Note}

Springer Nature remains neutral with regard to jurisdictional claims in published maps and institutional affiliations.

Received: 4 February 2020 Accepted: 24 April 2020 Published online: 07 May 2020

\section{References}

1. Masjed-Jamei, M.: A main inequality for several special functions. Comput. Math. Appl. 60, 1280-1289 (2010)

2. Maleševic, B., Lutovac, T., Rašajski, M., Mortici, C.: Extensions of the natural approach to refinements and generalizations' of some trigonometric inequalities. Adv. Differ. Equ. 2018, 90 (2018).

https://doi.org/10.1186/s13662-018-1545-7

3. Zhu, L., Hua, J.K.: Sharpening the Becker-Stark inequalities. J. Inequal. Appl. 2010, Article ID 931275 (2010). https://doi.org/10.1155/2010/931275

4. Sun, Z.J., Zhu, L.: Simple proofs of the Cusa-Huygens-type and Becker-Stark-type inequalities. J. Math. Inequal. 7(4), 563-567 (2013). https://doi.org/10.7153/jmi-07-52

5. Debnath, L., Mortici, C., Zhu, L.: Refinements of Jordan-Steckin and Becker-Stark inequalities. Results Math. 67, 207-215 (2015). https://doi.org/10.1007/s00025-014-0405-3

6. Lv, H.L., Yang, Z.H., Luo, T.Q., Zheng, S.Z.: Sharp inequalities for tangent function with applications. J. Inequal. Appl. 2017, 94 (2017). https://doi.org/10.1186/s13660-017-1372-5

7. Zhu, L.: Sharpening Redheffer-type inequalities for circular functions. Appl. Math. Lett. 22, 743-748 (2009). https://doi.org/10.1016/jaml.2008.08.012

8. Wu, S., Debnath, L.: A generalization of I'Hospital-type rules for monotonicity and its application. Appl. Math. Lett. 22, 284-290 (2009). https://doi.org/10.1016/.aml.2008.06.001

9. Yang, Z.H., Chu, Y.M., Wang, M.K.: Monotonicity criterion for the quotient of power series with applications. J. Math. Anal. Appl. 428(1), 587-604 (2015). https://doi.org/10.1016/j.jmaa.2015.03.043

10. Zhu, L:: New bounds for the exponential function with cotangent. J. Inequal. Appl. 2018, Article ID 106 (2018). https://doi.org/10.1186/s13660-018-1697-8

11. Banjac, B.D.: System for automatic proving of some classes of analytic inequalities. School of Electrical Engineering (2019). Available on: http://baig.etf.bg.ac.rs/

12. Malesevic, B., Makragic, M.: A method for proving some inequalities on mixed trigonometric polynomial functions. J. Math. Inequal. 3, 849-876 (2016)

13. Zhu, L., Malešević, B.: Natural approximation of Masjed-Jamei's inequality. Rev. R. Acad. Cienc. Exactas Fís. Nat., Ser. A Mat. 114, 25 (2020)

14. Chen, C.P., Malešević, B.: Inequalities related to certain inverse trigonometric and inverse hyperbolic functions. Rev. R. Acad. Cienc. Exactas Fís. Nat., Ser. A Mat. 114, 105 (2020)

15. Zhu, L., Maleševic, B.: Inequalities between the inverse hyperbolic tangent and the inverse sine and the analogue for corresponding functions. J. Inequal. Appl. 2019, 93 (2019) 